\title{
A midiatização em perspectiva crítica
}

\author{
Thales Vilela Lelo' \\ https://orcid.org/0000-0002-6070-9332 \\ I - Universidade de São Paulo. \\ São Paulo (SP). Brasil.
}

\begin{abstract}
Resumo: O artigo propõe uma revisão crítica da literatura europeia em midiatização, especificamente a vertente socioconstrutivista. O objetivo é evidenciar que as pesquisas aglutinadas nessa corrente têm frequentemente desconsiderado as contradições que permeiam o fenômeno da midiatização por idealizarem as transformações socioculturais decorrentes dele. São recuperados os principais argumentos de autores que abordam o conceito em perspectiva micro, meso e metaprocessual, assinalando suas lacunas teóricometodológicas e indicando outras chaves investigativas para apreender as ambivalências que atravessam as sociedades saturadas de mídia.
\end{abstract}

Palavras-chave: midiatização; socioconstrutivismo; crítica.

\begin{abstract}
A critical standpoint to mediatization approach - The article proposes a critical review of European literature of mediatization, specifically the social-constructivist approach. The main goal is to highlight that research in this field has often disregarded the contradictions that permeate mediatization by idealizing the sociocultural transformations resulting from it. The central arguments of authors who approach the concept on micro, meso, and meta-level are recovered, pointing out its theoretical-methodological gaps and highlighting other fruitful investigative keys to grasping the ambivalences that cross media-saturated societies.
\end{abstract}

Keywords: mediatization; social-constructivist approach; critics.

\section{Introdução}

A consolidação internacional dos estudos em midiatização caminhou em paralelo à percepção de que as atividades humanas são, contemporaneamente, "saturadas" pela mídia. Se o reconhecimento dessa ubiquidade das 
tecnologias de informação e comunicação nos mundos sociais possibilitou um progresso em face das clássicas teorias da comunicação de massa, como pontua Livingstone (2009), é oportuno também destacar que, em seu emprego recente, o conceito de midiatização foi utilizado, no transcorrer dos anos de 1980, visando descrever a incidência da lógica dos meios na esfera política, como salientam Hjarvard (2012) e Ampuja, Koivisto e Väliverronen (2014).

As discussões acerca das especificidades do fenômeno da midiatização se tornaram gradualmente influentes nos estudos em Comunicação nos anos 2000, sobretudo no que concerne à sua devida formulação teórica. Se, para investigadores como Hjarvard, o conceito resguarda certa dinâmica causal ao privilegiar a penetração inercial das formas da mídia em espaços da vida contemporânea, isto é, "o processo pelo qual a sociedade, em um grau cada vez maior, está submetida a ou torna-se dependente da mídia e de sua lógica" (2012, p. 64); para um conjunto ainda mais expressivo de pesquisadores, o programa de estudos em midiatização se consolida na investigação das inter-relações difusas e de longo prazo entre as mudanças em escopo sociocultural e aquelas propriamente tecnocomunicacionais.

Não obstante os debates supracitados sejam relevantes para o desenvolvimento dos estudos em midiatização, considera-se que é o momento de refletir acerca de inconsistências até então pouco levadas em consideração por alguns desses trabalhos. Destarte, distintamente dos empreendimentos de revisão literária que se dedicam a cartografar as nuances do fenômeno da midiatização recorrendo à etimologia do termo, às suas relações com conceitos correlatos (como mídia, meio e mediação) ou aos seus primeiros usos na história, este artigo se enfoca na vertente socioconstrutivista, bastante influente na Europa (COULDRY; HEPP, 2013)1', com o intuito de problematizar certo déficit crítico nessa bibliografia², que tem conferido atenção privilegiada ao mapeamento das transformações socioculturais decorrentes da apropriação criativa de dispositivos sociotécnicos em detrimento da detecção e problematização das iniquidades (em suas mais diversas formas de manifestação) envolvidas nas lógicas da midiatização³.

1 Tal agenda investigativa também passou a exercer influência no contexto latino-americano, como assinala Rüdiger (2015). Contudo, a trajetória do conceito na região remonta ao final dos anos de 1980, com a perspectiva semioantropológica de Eliseo Verón - que se distingue da abordagem socioconstrutivista europeia.

2 A bibliografia de referência a este estudo toma por base publicações precedentes que se dedicaram a elencar os principais autores da tradição socioconstrutivista, a exemplo de Adolf (2017), Ampuja, Koivisto e Väliverronen (2014), Couldry e Hepp (2013) e Lunt e Livingstone (2015).

3 As críticas que serão aqui formuladas poderiam ser também estendidas à perspectiva institucionalista dos estudos em midiatização, uma vez que a argumentação de Hjarvard (2012) pressupõe, em linhas gerais, a mesma naturalidade à gradual midiatização da sociedade quando trata da institucionalização da mídia e de sua incidência nas demais instituições sociais. 
É importante ressaltar, de partida, que os questionamentos delineados ao longo deste artigo não buscam invalidar os relevantes subsídios teórico-metodológicos oferecidos pela perspectiva socioconstrutivista (distinguindo-se, portanto, das críticas cáusticas ao conceito feitas por Deacon e Stanyer (2014) e Rüdiger (2015)). Como recentemente ressaltado por Lunt e Livingstone (2015) e Livingstone (2019), se em termos teóricos essa literatura possibilitou reconsiderar as transformações que experimentamos em nosso ecossistema midiático sem perder de vista o seu enraizamento sociocultural, em aspecto institucional possibilitou a confluência de investigações que permaneceriam dispersas não fossem seus arcabouços teóricos e instrumentos metodológicos. Dessa forma, o que se visa reivindicar neste estudo é uma dimensão normativa aos trabalhos que adotem a midiatização como conceito-chave, isto é, uma preocupação crítica com a face canhestra de um processo que se insere em sociedades marcadas por assimetrias. Considera-se que seja o momento de reclamar maior equilíbrio entre a inclinação descritiva que perpassa a bibliografia socioconstrutivista (como será discutido adiante) e uma ênfase analítica nas consequências, por vezes adversas, do fenômeno da midiatização. Trata-se, enfim, de uma crítica interna ao conceito que recorre a algumas de suas reformulações recentes no intuito de contribuir para seus futuros desdobramentos e apropriações.

Na primeira seção, serão recuperados os argumentos mais correntes da tradição de pesquisa supracitada, de modo a questionar certa inclinação desses trabalhos em superestimar a engenhosidade da agência humana no uso das tecnologias para a resolução de problemas socialmente construídos. Busca-se ressaltar que as dinâmicas interacionais midiatizadas podem reiterar mecanismos de exploração e amplificar expressões de intolerância e discriminação. Assevera-se também que a onipresença das tecnologias de informação e comunicação na sociedade não pressupõe uma transformação necessariamente benigna das formas de construção da realidade sociocultural, uma vez que tal processo é conformado por estratégias de regulação econômica que afluem na dependência das gramáticas de reconhecimento provenientes do universo midiático.

Já o segundo tópico é dedicado a uma vertente adjacente da literatura socioconstrutivista, que investe em uma teleologia histórica da midiatização, caracterizada como componente intrínseco ao desenvolvimento humano. O idealismo impresso nessa proposição será questionado à vista da infraestrutura de opressões e trabalhos precários que viabilizam a expansão 
global das tecnologias no capitalismo. Em última instância, propõe-se uma agenda investigativa que reconheça o caráter espacialmente referenciado do fenômeno da midiatização, uma vez que a paulatina incorporação de novos dispositivos técnico-comunicacionais configura um processo ambivalente, que distribui desigualmente suas benesses à sociedade.

\title{
O que se oculta na midiatização?
}

Couldry e Hepp (2013) cartografam o surgimento da agenda de pesquisa em midiatização na literatura europeia a partir dos anos 2000. Reconhecendo as origens do conceito, eles distinguem uma vertente institucionalista (ainda bastante dependente das conceituações provenientes dos anos de 1980, e tendo Hjarvard como seu principal expoente), de uma perspectiva socioconstrutivista, a qual os responsáveis pelo editorial não só se identificam, mas assinalam como um patente avanço diante da literatura existente. Em suas palavras, de um prisma socioconstrutivista, o conceito de midiatização

\begin{abstract}
capturaria tanto como a construção comunicativa da realidade se manifesta a partir de certos processos midiáticos e como, por sua vez, características específicas de certas mídias têm uma 'consequência' contextualizada para o processo geral pelo qual a realidade sociocultural é construída na e por meio da comunicação (COULDRY; HEPP, 2013, p. 196) ${ }^{4}$.
\end{abstract}

Esta conceituação reaparece, com ligeiras modificações, nos textos de diversos outros pesquisadores que reivindicam a mesma filiação teórica. Para Knoblauch (2013), por exemplo, “o estudo da midiatização é, portanto, o estudo da transformação da estrutura da ação comunicativa" (KNOBLAUCH, 2013, p. 310). Já Hepp e Krotz (2014) sugerem que a abordagem socioconstrutivista ofereceria um horizonte não midiacêntrico para os estudos em comunicação, por possibilitar "uma análise crítica da interrelação entre as mudanças na mídia e na comunicação, por um lado, e as mudanças na cultura e na sociedade, por outro" (HEPP; KROTZ, 2014, p. 3). Os autores ainda propõem que a chave heurística para essa conceituação é a observação empírica de fragmentos do mundo social, progressivamente articulados às redes de comunicação mediada (alcunhados de mundos midiatizados).

A seu turno, Andersson (2017) e Hepp e Hasebrink (2015) reivindicam uma visada empírica análoga para os estudos em midiatização: enquanto 
o primeiro sugere que "o melhor pré-requisito para apreender o significado da mídia na vida cotidiana - ou seja, as camadas matizadas da midiatização - é focar na vida cotidiana, e não na mídia" (ANDERSSON, 2017, p. 48), os segundos propõem averiguar, na concretude das situações (ou "configurações comunicativas", em sua terminologia), os sensíveis deslocamentos nos padrões de interação simbólica derivados da propagação moderna de dispositivos sociotécnicos. Aqui, as formas mais institucionalizadas da midiatização, concebidas enquanto mídias (televisão, rádio etc.), são tão somente formatos estabelecidos e permanentemente reconfigurados à luz das interações que se desenham através delas.

Não obstante a autodesignação dessa agenda de pesquisas sobre a midiatização como socioconstrutivista seja objeto de disputa nas revisões de literatura produzidas na Europa (AMPUJA; KOIVISTO; VÄLIVERRONEN, 2014; ADOLF, 2017), tais autores compartilham uma visão socioinstrumental da mídia enquanto comunicação, ou seja, "por esta perspectiva, a comunicação toma precedência sobre a mídia, cuja particularidade como tecnologia material é usualmente posta de lado em prol de uma ênfase na importância da agência humana ou da prática institucional" (ADOLF, 2017, p. 24). Por essa razão, nesses trabalhos é saliente uma inclinação à idealização epistêmica da agência humana como instância inerentemente reguladora das tecnologias em benefício à transformação sociocultural ${ }^{5}$, tendência que acaba por interditar uma discussão crítica dos pressupostos teóricos subjacentes à abordagem socioconstrutivista. Não surpreende, portanto, que os autores alinhados a essa corrente privilegiem o desenvolvimento de conceitos operadores para o estudo empírico da midiatização6 .

Mas, assim como é possível discorrer sobre circunstâncias nas quais a paulatina midiatização da sociedade viabiliza uma agência criativa capaz de reconfigurar as tecnologias disponíveis ou mesmo apropriá-las visando o progresso humano, é também bastante razoável discutir processos de experimentação igualmente engenhosos, mas que servem à normalização ou ao recrudescimento das assimetrias sociais existentes. Investindo criticamente no contexto da gig economy, Chan e Humphreys (2018) analisam como

5 Knoblauch (2013), por exemplo, assegura que, devido ao fato de a midiatização permitir a consecução da ação comunicativa em ambientes descontextualizados (reduzindo à relevância das relações sociais espacialmente situadas), ela necessariamente levaria a uma reconfiguração da comunidade e do espaço público (em prol de uma sociabilidade ampliada).

6 Preocupação que inclusive mobilizou, na Europa, a elaboração das noções de "mundos midiatizados" (KROTZ; HEPP, 2014) e de "configurações comunicativas" (HEPP; HASEBRINK, 2015). 
os motoristas de Uber interpretam o espaço social midiatizado que percorrem diariamente por meio do aplicativo. Conforme os autores, por meio de fóruns virtuais (como o Uber People), esses trabalhadores tentam atribuir sentido aos herméticos algoritmos de avaliação e remuneração da empresa que normatizam sua atividade sem que eles tenham o poder de entendê-los ou questioná-los abertamente. Destarte, desenvolvem táticas para antecipar pontos na cidade que possam vir a se converter em rotas de "tarifa dinâmica" (onde a maior demanda por motoristas gera um aumento nos valores das corridas); aprendem como se vestir e a se portar diante dos passageiros para garantir uma avaliação alta, e também sobre quais clientes devem evitar para não receberem notas ruins. Logo, a "inventidade" social dos usuários do fórum, antes de ser um ato de resistência contra o sistema gestionário da Uber, restringe-se a uma normalização de seus mecanismos de controle:

Embora os motoristas sejam duros em suas críticas ao sistema de classificação, eles o legitimam atribuindo significados sociais [...] a passageiros com uma nota abaixo do padrão. Isto é, este sistema se torna uma forma de disciplina baseada em dados que modelam a maneira como os motoristas interagem com a plataforma e os passageiros (CHAN; HUMPHREYS, 2018, p. 34).

Mas, mesmo se o escopo for ampliado para outros setores da gig economy (WOOD et al., 2018), casos similares de reiteração das desigualdades, quando da apropriação de novos dispositivos sociotécnicos, são facilmente identificáveis. No caso de entregadores de aplicativos que atuam em grandes centros urbanos, é comum que eles aceitem bônus financeiros das empresas para permanecerem em determinadas regiões por longos períodos de tempo, o que pode motivar alguns a dormirem nas ruas para emendarem horários de pico de pedidos sem terem de voltar para casa, compondo uma jornada diária superior a 12 horas ininterruptas ${ }^{7}$. Além disso, em países que recebem alto volume de imigrantes ilegais (como na Inglaterra e na Irlanda), há um mercado informal de anúncios (presente em fóruns de redes sociais como o Facebook e o Whatsapp) para locação de contas em aplicativos de entrega, voltado a pessoas sem passaporte ou comprovante de endereço (que são, por lei, vetadas de prestarem serviços para essas empresas) ${ }^{8}$.

7 Disponível em: <https://www.bbc.com/portuguese/brasil-48304340>. Acesso em: 13 set. 2019.

8 Disponível em: <https://g1.globo.com/mundo/noticia/2019/07/24/o-mercado-ilegal-das-contas-de-app-de-entrega-alugadas-por-brasileiros-em-londres.ghtml>. Acesso em: 13 set. 2019. 
Mas, para além da normalização de assimetrias no mundo do trabalho em decorrência da gradual midiatização da sociedade, em outras esferas da realidade, a ativa experimentação das tecnologias pode atender também a finalidades antidemocráticas - que desembocam em uma miríade de formas de intolerância e desrespeito. Pesquisadores como Peters (2015) e Krzyżanowski (2018) vêm empregando uma leitura crítica do conceito de midiatização para assinalarem como a instrumentalização dos potenciais de participação das novas mídias oportuniza a vocalização de uma agenda conservadora e nazifascista que se fortaleceu na Europa nos últimos anos. Para Peters, que estudou ações da rede neofascista The Immortals na Alemanha,

os usos das mídias sociais e das tecnologias de comunicação em rede não só afetaram a ação ou a forma de protesto do grupo, a qual foi desenhada para disseminar em várias plataformas do gênero, mas também modificou significativamente a estrutura organizacional e de mobilização, que pode ser caracterizada como uma rede flexível, não hierárquica e frouxa (2015, p. 149).

Já Krzyżanowski (2018), focado no contexto da política antimigratória polonesa e do crescente apoio ao populismo de extrema direita, averigua os deslocamentos discursivos empregados pelas autoridades locais em seus vídeos postados em plataformas de compartilhamento (como o Youtube) para construírem uma narrativa permeada por dados falsos, que atende ao propósito de instigar o preconceito contra refugiados (especialmente os muçulmanos, acusados de espalhar doenças e causar prejuízos econômicos ao país).

Essa leitura mais atenta às contradições que atravessam a "sociedade em midiatização", frequentemente negligenciada pelos socioconstrutivistas, alerta para os riscos de limitar o escopo de análise à descrição das experimentações sociais possibilitadas pelos novos dispositivos técnicos disponíveis. Isto é, as inter-relações entre as transformações nas mídias e no tecido sociocultural podem também ser mobilizadas para a compreensão de inflexões antidemocráticas nos "mundos midiatizados".

Complementarmente, caberia também problematizar a submissão, de certo modo, involuntária dos indivíduos às plataformas digitais controladas por corporações como o Google e o Facebook, que demandam dados pessoais daqueles que as acessam e instituem práticas de autoexposição dos usuários com a promessa de reconhecimento intersubjetivo. Autores como Jansson (2015) e Campanella (2019) vêm refletindo sobre como as mídias sociais se 
autopromovem difundindo um ideal de autorrealização intensamente buscado na modernidade para oferecer, em troca, formas ideológicas desse reconhecimento - formas essas que não tornam os indivíduos mais autônomos, mas sim cada vez mais dependentes de "um modelo moral que confere valor a identidades e práticas ligadas ao mundo da mídia" (CAMPANELLA, 2019, p. 2).

Desse prisma, o caráter pervasivo da midiatização se apresenta também como resultado de um controle econômico que contribui para a regulação da paisagem sociocultural. Conforme Jansson (2015), a normalização de uma série de novas rotinas diárias (como consultar em diversos momentos do dia os perfis em mídias sociais, ou capturar imagens tendo como propósito primeiro seu compartilhamento) e a naturalização da indispensabilidade de certos dispositivos (como smartphones e redes Wi-Fi), reforçam um imaginário de saturação midiática "que, por sua vez, reproduz a dependência funcional em relação a vários sistemas e infraestruturas tecnológicas" (JANSSON, 2015, p. 88).

Diante desses questionamentos, ao que a perspectiva socioconstrutivista reivindica como um programa investigativo para os estudos em midiatização, a próxima seção empreenderá uma crítica similar a outro conjunto de expoentes dessa literatura, que, em prisma socioevolutivo, conceituam as lógicas da midiatização como um evento intrínseco ao desenvolvimento da humanidade.

\section{Uma longa história de "saltos evolutivos" atravessada por exploração}

Ainda que frequentemente aglutinados sob a rubrica do socioconstrutivismo (COULDRY; HEPP, 2013), por também considerarem a midiatização como um fenômeno de construção interacional da realidade, alguns pesquisadores europeus se distinguem dos seus colegas por se referirem à mídia como a intrínseca medialidade presente na relação humana com o mundo, não se restringindo, portanto, aos dispositivos modernos que permitem a extensão da ação comunicativa. Conforme pontua Adolf (2017), tal especificidade pavimenta uma abordagem macroestrutural e antropológica da midiatização.

Por essa razão, os trabalhos que se alinham a essa conceituação se distinguem da outra vertente do socioconstrutivismo por compreenderem a "midiatização" como um metaprocesso de extensa duração histórica, duração essa que pode remontar à própria constituição da humanidade (KROTZ, 2007, 2014, 2017; SCHULZ, 2004). E, ainda que haja um forte acento no papel 
da tecnologia como forma que incide sobre as sociedades (em sentido similar ao proposto pela teoria do meio), percebe-se que os autores que propõem essa leitura de longo prazo da midiatização reivindicam uma espécie de "darwinismo social" a reger as transformações históricas ocorridas.

Schulz (2004), por exemplo, delineia um programa em quatro fases sequenciais que retrataria o desenvolvimento natural da midiatização na tessitura social: inicialmente, os limites da comunicação humana seriam expandidos por novos dispositivos técnicos; na sequência, as atividades de diversos atores e instituições seriam reconfiguradas; em seguida, as fronteiras entre atividades midiáticas e não midiáticas seriam dissolvidas; até que, por fim, tudo culminaria em uma acomodação integral às novas dinâmicas de interação. Krotz $(2007,2014,2017)$ também persegue essa trilha ao reclamar uma dimensão metaprocessual para o estudo da midiatização, entendida aqui em chave equivalente a outras noções correntes na teoria social, como a globalização, a individualização e a comercialização. Para o comunicólogo alemão, a midiatização é processo de longo prazo atrelado aos desenvolvimentos da linguagem e das capacidades de interação humana. Nesse sentido, a demanda por novas tecnologias atenderia às necessidades e interesses da sociedade em determinado momento histórico. Em suas palavras, "não é a diferenciação da mídia, a tecnologia ou a lógica midiática que altera as condições da comunicação. É simplesmente a comunicação: as pessoas utilizam diferentemente as mídias [...] e então se comunicam diversamente" (KROTZ, 2014, p. 83). Por essa chave explicativa, a emergência de dispositivos técnico-comunicacionais seria consequência natural de necessidades de simbolização não atendidas pelos recursos disponíveis em determinada época. A midiatização traduz-se, assim, em processo regulador intrínseco ao desenvolvimento social.

Essa celebração da midiatização decorre, em certa medida, do elevado grau de abstração dessas proposições e da negligência de dimensões geoespaciais e infraestruturais em sua elaboração. São esses esquecimentos que naturalizam, no plano epistêmico, o darwinismo presente nelas. Apesar de Krotz (2017) ter recentemente percebido essa desatenção ao propor uma conceituação crítica e progressista da midiatização, reconhecendo que o fenômeno retratado não segue um curso natural em favor dos parâmetros evolutivos da espécie humana - sendo, então, permeado pela dinâmica do capital e por jogos de poder que quase nunca admitem a ampla inserção da sociedade civil -, as suas ponderações são ainda bastante tímidas e se 
limitam a: a) consentir com o fato de que a infraestrutura digital é controlada por um número reduzido de corporações multinacionais que atendem a interesses estritamente econômicos; e b) admitir a existência de "barreiras digitais" que inviabilizam que certos grupos sociais possam usufruir das novas tecnologias.

Seria também imprescindível reconhecer que subjaz à emergência de novos dispositivos técnicos um circuito de exploração de mão de obra e de trabalhos precários. Circunscrevendo ao período histórico tematizado por Krotz em sua recente reconsideração crítica dos estudos em midiatização, seria importante pontuar, por exemplo, que a "apropriação social" das tecnologias de interação não se restringe aos seus usos criativos, mas também a uma dinâmica de atividades mecanizadas executadas por humanos que servem à otimização dos algoritmos empregados por assistentes digitais e motores de busca. Como relatam Wood et al. (2018), em sua investigação com trabalhadores da Ásia e da África Subsaariana que executam remotamente microtarefas para plataformas como a Amazon Mechanical Turk (MTurk), as remunerações mensais muitas vezes são inferiores aos salários mínimos de seus países de origem, não há proteções jurídicas e as jornadas atingem até 16 horas diárias, acarretando em isolamento social, privação de sono e exaustação.

Determinadas tarefas desempenhadas na "sociedade em midiatização" podem inclusive gerar, em curto prazo, danos consideráveis à saúde mental daqueles que as exercem. Por detrás das saudadas transformações socioculturais decorrentes da incorporação de novos dispositivos técnicos à ação humana, reside um contingente expressivo de trabalhadores mal pagos filtrando, via moderação de conteúdo, quaisquer materiais que representem violação aos termos das plataformas digitais. Atuando amiúde em firmas terceirizadas que prestam serviços para gigantes do setor, sem treinamento ou condições apropriadas de trabalho (sobretudo em países como as Filipinas e a Índia) e com remunerações insuficientes (que podem oscilar entre US\$ 1,00 e US\$ 4,00 por hora), esses sujeitos são expostos diariamente a uma torrente de dados detectados como impróprios por usuários ou pela identificação automática das plataformas. Como informa Gillespie (2018), tais conteúdos vão de "material inofensivo sinalizado acidental ou incorretamente, a coisas questionáveis que poderiam cruzar ou cruzam a linha, indo até um conjunto expressivo de atrocidades humanas" (GILLESPIE, 2018, p. 66). Segundo o autor, muitos moderadores têm, nessa atividade, sua única fonte de renda e podem vir a visualizar por volta de mil e quinhentas imagens por 
dia, incluindo pedofilia, decapitações e abuso de animais (além de expressões de violência mais cotidianas, como racismo, homofobia e sexismo). É frequente que esses trabalhadores sofram de ataques de pânico e de Transtorno de Estresse Pós-Traumático (TEPT), muito comum entre veteranos de conflitos armados. Ademais, as elevadas metas de precisão na moderação, impostas pelas empresas, atreladas a ambientes de trabalho pautados por rígidas métricas de desempenho, acarretam em uma enorme rotatividade de funcionários, que permanecem em média um ano no setor até serem demitidos ou se demitirem.

Mas, como também assinala Gillespie (2018), uma parcela do trabalho de moderação é exercida gratuitamente pelos próprios usuários, que podem ser convidados por redes sociais a classificarem postagens específicas ou seus próprios perfis nas plataformas. A propósito, conforme salientam Dantas, Canavarro e Barros (2014), o trabalho gratuito é um instrumento fulcral de acumulação de capital na "sociedade em midiatização", haja vista que, mesmo em redes colaborativas (como o Avaaz), "os dados informacionais quantitativos suscetíveis de serem submetidos a cálculos probabilísticos, extraídos do trabalho vivo dos ativistas, são aproveitados, pela Purpose ${ }^{9}$, para a prestação de serviços de consultorias para grandes empresas globais" (DANTAS; CANAVARRO; BARROS, 2014, p. 24).

Em linhas gerais, falta à perspectiva metaprocessual dos estudos em midiatização realizar um movimento dialético que abarque as contradições intrínsecas ao fenômeno tratado quando de sua inserção nas mais diversas comunidades humanas. Rendida a uma celebração dos potenciais transformadores da midiatização, tal literatura delineia um programa investigativo exclusivamente dedicado a descrever como este "salto evolutivo" representado pela midiatização se manifesta in $100^{10}$. Perde-se de vista, além das questões supracitadas, como a apropriação de dispositivos sociotécnicos pode também ser exercida verticalmente, como discorrem Dyer-Witheford (2016) acerca do desmantelamento do campesinato nos países periféricos e Huws (2011) sobre a "re-proletarização" de uma miríade de setores profissionais (indo do jornalismo aos serviços de contabilidade). Em decorrência da emergência de novas tecnologias de controle e metrificação do trabalho,

9 Organização sediada nos Estados Unidos que se propõe a "incubar" plataformas de mobilização em nível global e local.

10 Questão que também preocupa Deacon e Stanyer (2014) e Rüdiger (2015) em suas críticas a essa agenda de pesquisa. 
além da possibilidade de padronização de determinados processos, "tornou-se possível monitorar os trabalhadores pelos resultados, e realocar a operação em qualquer parte do globo onde a estrutura correta esteja disponível" (HUWS, 2011, p. 39).

Para encerrar esta seção, cumpre salientar que as consequências menos laureadas da midiatização, entendida como um metaprocesso, não se restringem ao momento presente. Poderíamos reconstruir o trajeto histórico de longa duração sugerido pelos autores que se afinam a essa perspectiva e identificar, antes de um paulatino desenvolvimento social da humanidade em decorrência da incorporação de novos dispositivos técnico-comunicacionais, um fenômeno canhestro, que correntemente beneficia determinados grupos à custa da exploração e opressão de outros. Como nos adverte Ampuja (2015), é inerente ao capitalismo a tendência de espraiar globalmente sua racionalidade, e a cobertura de vastas distâncias geográficas só é viável a partir da sofisticação de tecnologias de comunicação e transporte ${ }^{11}$. Mas, mesmo antes da Idade Moderna, se recuperarmos o exemplo dado por Krotz (2014) da cultura monástica do século XII como uma ilustração da historicidade da midiatização, chama atenção a força de dominação exercida pela Igreja (equivocadamente negligenciada pelo autor) ao circunscrever o acesso às obras bíblicas e filosóficas às autoridades que tinham o privilégio de serem alfabetizadas.

\section{Considerações finais}

Longe de reclamar uma dose de tecnofobia para os entusiastas da midiatização, o presente artigo procurou explicitar algumas lacunas na abordagem socioconstrutivista europeia no intuito de reivindicar uma guinada normativa aos trabalhos que se afinam a ela. Argumentou-se que, se os processos de midiatização estão inseridos no universo sociocultural, são sujeitos às mesmas contradições que o permeiam (o que pode inclusive contribuir, como foi demonstrado, para o recrudescimento da injustiça e para a reiteração de uma infraestrutura econômica calcada em explorações).

Como ressaltado ainda na introdução, a ênfase que este trabalho atribui a uma dialética da midiatização está em consonância a uma crítica feita por

11 Em outro artigo, Ampuja, Koivisto e Väliverronen (2014) também assinalam que, a despeito de Krotz (2007) ter reconhecido timidamente que no capitalismo todos os metaprocessos (incluindo a globalização, a individualização e a comercialização) têm por referência a ordem econômica vigente, essa consideração não gerou naquele momento verdadeiras inflexões nos estudos em midiatização. 
expoentes do próprio campo que não invalida a trajetória epistêmica do conceito e muito menos os aportes teórico-metodológicos fornecidos por ele até aqui. Recentemente, além de Krotz (2017), autores como Hepp e Hasebrink (2018) e Hepp (2020) vêm reconhecendo a necessidade de tratar do caráter perigosamente pervasivo dos algoritmos de coleta de dados e da hegemonia das plataformas digitais no desenvolvimento da infraestrutura tecnológica em um período de "midiatização profunda". Em tom muito menos vibrante, cada vez mais a literatura socioconstrutivista se interroga sobre a possibilidade de concretização da "vida boa" (HEPP, 2020) em condições de aprofundamento da midiatização - já reconhecendo que ela não é o destino inevitável do processo. Por essa razão, os questionamentos apresentados ao longo deste artigo aos pressupostos que subjazem ao arcabouço socioconstrutivista acenam para outros horizontes a serem desbravados pelas pesquisas da área em seus desdobramentos ulteriores, como sintetiza o quadro seguinte:

\begin{tabular}{|c|l|}
\hline Perspectiva micro e meso processual & $\begin{array}{l}\text { 1. } \begin{array}{l}\text { Normalização opressiva do espaço social } \\
\text { midiatizado. }\end{array} \\
\text { 2. Apropriações intolerantes e antidemocráticas } \\
\text { dos dispositivos sócio técnicos. }\end{array}$ \\
\hline Perspectiva metaprocessual & $\begin{array}{l}\text { Dataficação, dependência e reconhecimento } \\
\text { ideológico na sociedade midiatizada. }\end{array}$ \\
\hline $\begin{array}{l}\text { Formas de precarização e sofrimento que } \\
\text { permeiam a infraestrutura digital e econômica } \\
\text { da midiatização. } \\
\text { Distribuição geográfica desigual da exploração } \\
\text { nas sociedades midiatizadas. } \\
\text { Reestruturações produtivas decorrentes de uma } \\
\text { midiatização "verticalizada". }\end{array}$ \\
\hline
\end{tabular}

Quadro 1. Agenda de investigação para estudos críticos em midiatização. Fonte: elaboração própria.

Por fim, consideramos que essa agenda investigativa possibilita que os estudos em midiatização possam se dedicar mais incisivamente a uma análise das ambivalências que atravessam as sociedades saturadas pela mídia (tal qual sugere Jansson (2015)). Ou seja, trata-se de observar, nos meandros das interações paulatinamente impregnadas por dispositivos sociotécnicos, as iniquidades que delas podem emanar, descortinando não só suas incidências negativas na conformação da trama social, mas também engendrando formas de resistência a elas.

Thales Vilela Lelo é pesquisador de Pós-Doutorado na Escola de Comunicações e Artes da Universidade de São Paulo, com bolsa da Fapesp — Fundação de Amparo à 
Pesquisa do Estado de São Paulo. É doutor em Ciências Sociais pela Universidade Estadual de Campinas.

thales.lelo@gmail.com

\section{Referências}

ADOLF, M. The identity of mediatization: theorizing a dynamic field. In: DRIESSENS, O.; BOLIN, G.; HEPP, A.; HJARVARD, S. (Org). Dynamics of mediatization: institutional change and everyday transformations in a digital age. New York: Palgrave Macmillan, 2017. p. 11-34.

AMPUJA, M.; KOIVISTO, J.; VÄLIVERRONEN, E. Strong and weak forms of mediatization theory. Nordicom Review, v. 35, 2014. p. 111-123.

AMPUJA, M. A sociedade em rede, o Ccosmopolitismo e o "sublime digital": reflexões sobre como a história tem sido esquecida na teoria social contemporânea. Parágrafo, v. 1, n. 3 , 2015. p. 55-67.

ANDERSSON, M. Mediatization from below. In: DRIESSENS, O.; BOLIN, G.; HEPP, A.; HJARVARD, S. (Org). Dynamics of mediatization: institutional change and everyday transformations in a digital age. New York: Palgrave Macmillan, 2017. p. 35-56.

CAMPANELLA, B. Em busca do reconhecimento midiático: a autorrealização do sujeito na sociedade midiatizada. E-Compós, v. 22, n. 1, 2019. p. 1-19.

CHAN, N.; HUMPHREYS, L. Mediatization of social space and the case of uber drivers. Media and Communication, v. 6, n. 2, 2018, p. 29-38.

COULDRY, N.; HEPP, A. Conceptualizing mediatization: contexts, traditions, arguments. Communication Theory, v. 23, 2013. p. 191-202.

DANTAS, M.; CANAVARRO, M.; BARROS, M. Trabalho gratuito nas redes: de como o ativismo de $99 \%$ pode gerar ainda mais lucros para $1 \%$. Liinc em Revista, v. 10, n. 1, 2014. p. 22-43.

DEACON, D.; STANYER, J. Mediatization: key concept or conceptual bandwagon? Media, Culture and Society, v. 36, n. 7, 2014, p. 1032-1044.

DYER-WITHEFORD, N. Cybernetics and the making of a global proletariat. The Political Economy of Communication, v. 4, n. 1, 2016. p. 35-65.

GILLESPIE, T. Custodians of the internet: platforms, content moderation, and the hidden decisions that shape social media. New Haven \& London: Yale University Press, 2018.

HEPP, A.; KROTZ, F. Mediatized worlds - understanding everyday mediatization. In: HEPP, A.; KROTZ, F. (Org). Mediatized worlds: culture and society in a media age. New York: Palgrave Macmillan, 2014. p. 19-37.

HEPP, A.; HASEBRINK, U. Interação humana e configurações comunicativas: transformações culturais e sociedades midiatizadas. Parágrafo, v. 2, n. 3, 2015, p. 75-89. 
Researching Transforming communications in times of deep mediatization: a figurational approach. In: HEPP, A.; BREITER, A.; HASEBRINK, U. (Orgs.). Communicative figurations: transforming communications in times of deep mediatization. New York: Pallgrave Macmillan, 2018. p. 15-50.

HEPP, A. Deep mediatization. New York: Routledge, 2020.

HJARVARD, S. Midiatização: teorizando a mídia como agente de mudança social e cultural. MATRIZes, n. 2, 2012. p. 53-91.

HUWS, U. Mundo material: o mito da economia imaterial. Mediações, v. 16, n. 1, 2011, p. 24-54. JANSSON, A. Interveillance: A New Culture of Recognition and Mediatization. Media and Communication, v. 3, n. 3, 2015, p. 81-90.

LIVINGSTONE, S. Audiences in an age of datafication: critical questions for media research. Television \& New Media, v. 20, n. 2, 2019. p. 1-14.

LUNT, P.; LIVINGSTONE, S. Is 'mediatization' the new paradigm for our field? A commentary on Deacon and Stanyer $(2014,2015)$ and Hepp, Hjarvard and Lundby (2015). Media, Culture and Society, v. 38, n. 3, 2016. p. 1-9.

KNOBLAUCH, H. Communicative constructivism and mediatization. Communication Theory, v. 23, 2013. p. 297-315.

KROTZ, F. The meta-process of "mediatization" as a conceptual frame. Global Media and Communication, v. 3, n. 3, 2007. p. 256-260.

. Media, mediatization and mediatized worlds: a discussion of the basic concepts. In: HEPP, A.; KROTZ, F. (Orgs.). Mediatized worlds: culture and society in a media age. New York: Palgrave Macmillan, 2014. p. 72-90.

Explaining the mediatisation approach. Journal of the European Institute for Communication and Culture, 2017, p. 1-16.

KRZYZANOWSKI, M. Discursive shifts in ethno-nationalist politics: on politicization and mediatization of the "refugee crisis" in Poland. Journal of Immigrant \& Refugee Studies, v. 16, n. 1-2, 2018. p. 76-96.

LIVINGSTONE, S. On the mediation of everything. Journal of Communication, v. 59, n. 1, 2009. p. 1-18.

PETERS, R. Become immortal! Mediatization and mediation processes of extreme right protest. Conjunctions, v. 2, n. 1, 2015. p. 133-152.

RÜDIGER, F. Os estudos de mídia e a problemática epistemológica da teoria da midiatização - uma nova escolástica? Intexto, v. 34, n. 3, 2015. p. 745-769.

SCHULZ, W. Reconstructing mediatization as an analytical concept. European Journal of Communication, v. 19, n. 1, 2004. p. 87-101. 
WOOD, A.; GRAHAM, M.; LEHDONVIRTA, V.; HJORTH, I. Good gig, bad gig: autonomy and algorithmic control in the global gig economy. Work, Employment and Society, 2018, p. 1-20.

Artigo recebido em 01/06/2020 e aprovado em 14/08/2020. 